\title{
Operational Map-Guided Classification of SAR Sea Ice Imagery
}

\author{
Philippe Maillard, David A. Clausi, Senior Member, IEEE, and Huawu Deng, Member, IEEE
}

\begin{abstract}
This paper presents a map-guided sea ice classification system built to work in parallel with the Canadian Ice Service (CIS) operations to produce pixel-based ice maps that complement actual "egg code" maps produced by CIS. The system uses the CIS maps as input to guide classification by providing information on the number of ice types and their final label for specific regions. Segmentation is based on a modified adaptive Markov random field (MRF) model that uses synthetic aperture radar (SAR) intensities and texture features as input. The ice type labeling is performed automatically by gathering evidences based on a priori information on one or two classes and deducing the other labels iteratively by comparing distributions of segments. Three methods for comparing the segment distributions (Fisher criterion, Mahalanobis distance, and Kolmogorov-Smirnov test) were implemented and compared. The system is fully described with special attention to the labeling procedure. Examples are presented in the form of two CIS SAR-based ice maps from the Gulf of Saint Lawrence region and one example from the Beaufort Sea. The results indicate that when the segmentation is good, the labeling attains best results (between $71 \%$ and $89 \%$ ) based on evaluation by a sea ice analyst. Some problems remain to be assessed which are primarily attributable to discrepancies in the information provided by the egg code and what is actually visible in the SAR image. Subscale information on floe size and shape available to human analysts, but not in this classification system, also appear to be a critical information for separating some ice types.
\end{abstract}

Index Terms-Classification, distribution comparison, Fisher, gray-level cooccurrence matrix (GLCM), Kolmogorov-Smirnov, Mahalanobis, mapping, Markov random field (MRF), sea ice, segmentation, texture.

\section{INTRODUCTION}

$\mathbf{S}^{\mathrm{E}}$ EA ice recognition and mapping is a major operational application of remote sensing using orbital synthetic aperture radar (SAR). At the Canadian Ice Service (CIS), many SAR images are interpreted daily to produce sea ice charts that are sent to coast guard and merchant ships in sea-ice-infested regions for tactical route planning. Radarsat-1 with its horizontally copolarized $(\mathrm{HH}) \mathrm{C}$-band $(5.3 \mathrm{GHz}$ or $\lambda 5.7 \mathrm{~cm})$ imager is the primary source of data for CIS. The ScanSAR wide mode pro-

Manuscript received December 21, 2004; revised April 28, 2005. This work was supported in part by the Canadian Ice Service, in part by Geomatics for Informed Decisions (GEOIDE), and in part by the Cryosphere System in Canada (CRYSYS).

P. Maillard is with the Universidade Federal de Minas Gerais, Belo Horizonte, Brazil and also with the Department of Systems Design Engineering, University of Waterloo, Waterloo, ON N2L 3G1, Canada (e-mail: philippe@cart.igc.ufmg.br).

D. A. Clausi is with the Department of Systems Design Engineering, University of Waterloo, Waterloo, ON N2L 3G1, Canada.

H. Deng is with PCI Geomatics, Richmond Hill, ON L4B 1M5, Canada.

Digital Object Identifier 10.1109/TGRS.2005.857897 vides a swath of $500 \mathrm{~km}$ and can supply the necessary daily coverage of Canadian territory. The ice analysts use a geographic information system (GIS)/image processing (IP) environment to correct, enhance, interpret, and classify these images, but make no use of automated segmentation/classification techniques. Instead, they manually draw regions containing roughly homogeneous concentrations of sea ice types and open water for which they assign a code symbol called an egg code containing information on these types, their concentration, and ice floes sizes (http://ice-glaces.ec.gc.ca). These "egg coded" maps adopt the World Meteorological Organization (WMO) standard and are considered essential for navigation in sea-ice-infested regions. This interpretation work is done manually because there are no known effective operational tools available [1]. This can be partly explained by the fact that single-band SAR imagery is not easily processed using standard remote sensing segmentation and classification routines. The time factor is also an issue at CIS since the ice analyst interprets an image in less than an hour (D. Flett, personal communication, October 2004), a prohibitive time frame for many computer vision approaches.

Deng and Clausi [2] have designed and implemented a system that generates an unsupervised pixel-based segmentation of a SAR image based on an a priori estimation of the number of classes and using both texture and intensity features. A solution based on given homogeneous regions and a known number of sea ice classes is more likely to comply with WMO standards and to be adopted by an operations service. This study demonstrates the integration of these approaches into an operational environment. Whereas other systems rely solely on intensity and/or require training, MAGSIC was conceived to integrate texture with tone and to be unsupervised. Furthermore, an original solution for the labeling problem using classification on accumulated evidences is described and tested. This map-guided approach can be regarded as a symbiosis between machine-aided interpretation and ice analyst: while the first will benefit from a simplification of the problem and a higher chance of success, the second will see their work taken one step further and obtain more precise statistics on the sea ice types and open water percentages. At present, it would be impossible for CIS analysts to have the time to produce any pixel-based map of the ice types and a system that would perform such task would represent a meaningful progress for CIS operations (R. De Abreu, personal communication, May 2004).

Section II gives a background on the concepts adopted in our research and a short review of previous work that lead to the present paper. Section III describes the complete system and its components. Examples are presented in Section IV while Section V contains the conclusion. 


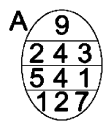

Fig. 1. Egg code symbol example. The letter "A" indicates that the symbol applies to region " $\mathrm{A}$ " in the scene. The first row indicates the total proportion of ice ( 9 indicates $90 \%$ ). The second row indicates the proportion of each ice type ( 3 in this case). The third row gives the ice code of each type, and finally the last row indicates the relative floe size for each ice type (http://ice-glaces.ec.gc.ca).

\section{BACKGROUND}

\section{A. Ice Services and Ice Maps}

Information on ice concentration, ice type, and movement is essential for the efficiency and safety of shipping as most navigable waters in Canada are infested with sea ice during at least the winter months for the southern part and year-round for the Arctic [3]. Ships navigating in these ice-congested seas must have timely and reliable information. Sea ice also has a direct effect on the climate, and global climate studies need to evaluate the quantity and proportions of open water, first-year, and multiyear ice in their models [4]-[6]. The advent of satellite SAR data with rapid return capacity has improved the ability to study sea ice and to produce ice maps in an operational manner [1], [6]. ${ }^{1}$ The Radarsat Geophysical Processor System (RGPS) brought a significant contribution to understanding the physics of ice backscattering and its relation to ice age, thickness, and deformation [7], [8], but more research still needs to be done in understanding the behavior of sea ice in SAR imaging. For example, Brown et al. [9, p. 68] point out that one of the "Two to Ten Year Action Items" is the continuation of the development of automated procedures at CIS to estimate geophysical parameters from Radarsat. At present, many ice services (i.e., Canada, U.S., Northern Europe) prepare WMO standard egg code maps that show sea ice information in the form of "homogeneous" regions having determined amounts (percentage) of different categories of ice (14 in all). ${ }^{2}$ Users of the egg code maps are then able to translate these ice categories into relative thickness [4]. Even though these maps are highly generalized, they are the expression of many years of experience in sea ice identification from SAR data and represent an invaluable source of information on which many lives and equipment rely. A sample egg code symbol is shown in Fig. 1. Although most of the data used are digital and mapping is done in a computer environment, these operations are still wholly based on manual interpretation, and the lack of automated algorithms for ice parameters retrieval has already been identified by ice services as a limiting factor in the quality of the information produced [6], [10]. The true challenge facing the different ice monitoring centers around the globe involves shifting from manual observation to automated algorithms, which in turn leads to improved prediction. More immediate challenges include the validation and improved access from new sensors and the improvement of information products for the end user [1]. The present paper is primarily concerned with the latter. In particular, the production of a full-resolution thematic ice map would lead to an improved

\footnotetext{
${ }^{1}$ See Ramsay et al. "Utilization of RADARSAT Data in the Canadian Ice Service" http://www.ccrs.nrcan.gc.ca/ccrs/rd/apps/.

${ }^{2}$ http://www.cis.ec.gc.ca/home.html
}

comprehension of a particular ice situation and would provide more precise statistical calculation of ice type proportions.

\section{B. Remote Sensing of Sea Ice Using Microwave Sensors}

Remote sensing of sea ice has been an area of active research for the last 15 years [4]. Because of frequent cloud coverage and polar winter darkness, optical remote sensing is often not usable for sea ice studies. Microwave sensors, being relatively unaffected by these factors, have become the primary source of data for sea ice remote sensing [3]. Since 1978, satellite-borne passive microwave sensors such as the Scanning Multichannel Microwave Radiometer, or the follow-up Special Sensor Microwave/Imager, have provided a continuous source of data that made possible small-scale (coarse resolution) quantitative estimates of the extent, nature (mainly multiyear versus first-year), and trends in the sea ice in the polar regions [11], [12]. Since the launch of a series of satellite-based SAR sensors (e.g., European Remote Sensing 1 and 2 satellites, the Japanese Earth Resources Satellite, and Radarsat-1), SAR has become the main source of data for operational detailed recognition and mapping of sea ice. Radar sensors have the additional advantage of being able to penetrate the ice surface and provide information on the ice structure and composition (mainly moisture and salinity) as well as being sensitive to the surface roughness [3], [13]. SAR backscattering is a function of two main categories of factors: electrical (dielectric constant) and geometrical (incidence angle and surface roughness). However, since these factors can vary simultaneously, predictive models, which makes attempts to infer geophysical surface parameters from the scattering values highly underdetermined [14]. Surface roughness is a function of wavelength, polarization, and incident angle; the fact that the latter varies within a single image is an additional complicating factor. The variation of the incidence angle can be modeled, but it remains a difficult task since different ice types react differently to such variation [15]. Each image pixel in a radar scene is also subject to constructive or destructive interference that leads to substantial variations of backscatter known as speckle or speckle noise [16]. Even when reduced using filtering techniques, speckle noise can be a strong undermining effect [14]. However, when taken in the context of visual texture analysis, speckle can bring benefits to the interpretation task [16], [17].

\section{Sea Ice Segmentation}

Other research efforts toward computer-aided interpretation of sea ice in SAR images have been attempted. The "Multi-year Ice Mapping System" (MIMS) was developed at the University of Colorado by Fetterer et al. [18] for the rapid identification of multiyear ice based on a local dynamic thresholding of SAR data using the Fisher criterion to split the distribution. Soh et al. [19] proposed an automated analysis of a sea ice system based on the Dempster-Shafer belief theory for segmentation and an expert system for classification. The system first performs a segmentation (based on the watershed merging algorithm) of the SAR image and then creates a series of 25 features that describes each segment. The features describe attributes of the segment such as average intensity (and other first-order statistics) as well as shape (and other spatial characteristics). Each segment is then 
classified according to a set of rules drawn from the knowledge base. Karvonen [20] developed a system based on a pulse-coupled neural network (PCNN). Training is based on the assumption that ice classes have a nearly Gaussian distribution, and since these distributions overlap, every pixel (within a window) can be modeled as belonging to some or all ice classes by detecting peaks (modes) in the distribution of the image tones. These $m$ classes are fed in the PCNN, which assigns the final label based on a neighborhood analysis.

\section{Texture}

Texture or visual texture has received much attention from the computer vision and remote sensing communities in the past 10 to 15 years (see [21] for a review). In the realm of texture representation, the gray-level cooccurrence matrix (GLCM) [22] is the most commonly used texture method in remote sensing applications [23]. The method has proven very powerful in a variety of remote sensing applications with optical data [24], [25] as well as for sea ice using SAR data [5], [26]-[28]. More specifically, the GLCM approach can accurately capture the textural characteristics of sea ice using SAR data [29] and has often proven superior to other popular methods in a classification context [24], [30]. The method is based on measurements taken from the matrix formed by the joint probability of a pair of gray level values at a fixed distance and orientation from each other. A wealth of measurements can be taken from the GLCM; Haralick et al. [22], being recognized as the first to use the GLCM in image processing, have proposed 14 measurements. Many of these are correlated or even redundant, and the past three decades of experience with GLCM has led to the usage of set of measurements that have been generally more successful: contrast and entropy in the present case [28], [31].

\section{E. Markov Random Fields for Segmentation}

Markov random fields (MRFs) can provide solutions for most contemporary image analysis problems such as image restoration, texture description, and image segmentation [32], [33]. MRF models inherently describe spatial context: the local spatial interaction among neighboring pixels. This is most appropriate since neighboring pixels are generally not statistically independent but are linked by spatial correlation. The Markov assumption states that the conditional probability of a pixel value given its neighborhood is equal to the conditional probability of that pixel given the rest of the image. In other words, each pixel with its local neighborhood can be considered an independent process making it more easily modeled in mathematical terms [34]. Other advantages of MRF models are that they can be inferred in the Bayesian framework and that they can work with multiple features (intensity and texture in the present case). Numerous MRF-based segmentation methods have been developed [2], [35]-[38]. Considering SAR images, MRF models have already shown to provide an appropriate representation of SAR images given their variance (due to speckle) and texture [38]-[41].

In this paper, the standard form of the MRF segmentation model adopted is described in Li [33] and adapted by Deng and Clausi [2]. The model named "modified adaptive Markov random field segmentation" (MAMSEG) uses the Bayesian

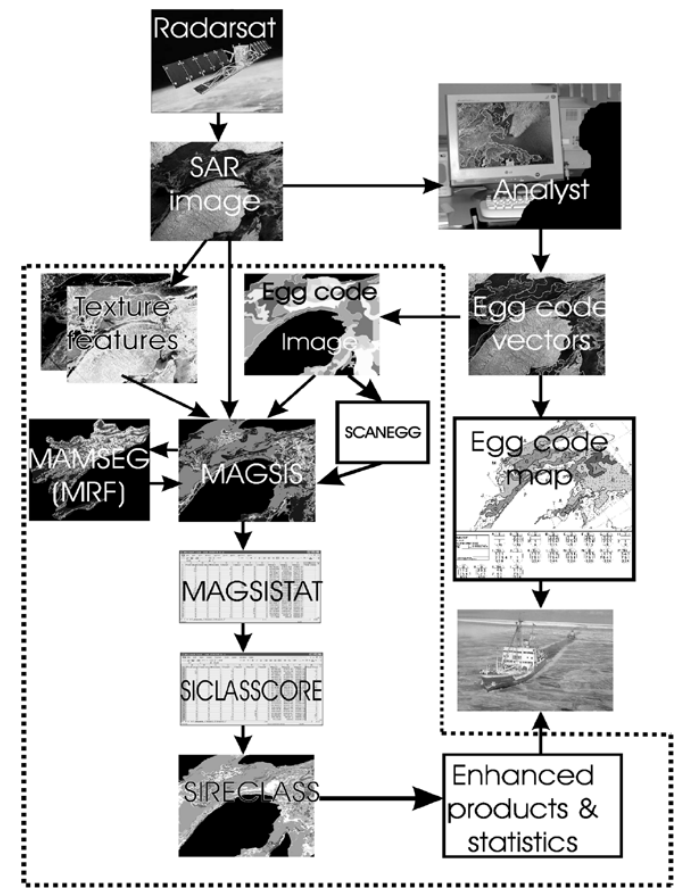

Fig. 2. Schematical representation of the system architecture (within the dotted line) and its relation with CIS operations (Radarsat-1 illustration, courtesy Canadian Space Agency-CSA).

paradigm where the conditional probability is based on a single observation (pixel vector) and a particular distribution (Gaussian or Gamma), and the prior probability is modeled using the neighborhood and the MRF model. The relationship between these two components is usually pre-fixed, but the current implementation introduced a variable weighting factor between them.

\section{Description Of THE System}

The system is not meant to be eventually inserted in the CIS operations but rather to operate in parallel to the CIS procedure and eventually provide value-added products such as thematic maps and improved statistics that are not feasibly produced by human operators due to time constraints. Both operations and their relationship are roughly illustrated in Fig. 2. The data used by the system consist of three files, namely: the SAR image (Radarsat-based), the vector-based egg code map file, and its attribute file containing the egg code information for each polygon in the egg code map. These data files are described below.

\section{A. Data and Preprocessing}

The area enclosed in the dotted line in Fig. 2 represents the MAp-Guided Sea Ice Classification (MAGSIC) system which can be activated by supplying the sea ice analyst's interpretation (in the form of a vector map and an attribute file) along with the registered SAR image. The vector map is supplied in the form of a "topologically clean" set of polygons (lines intersect where they are expected to; nodes are created at all intersections; all polygons are closed and contain a label; and no lines are duplicated [42, p. 195]) and the attribute file as a spreadsheet. At present, these files are prepared on a one-by-one basis using PCI Geomatica (Richmond Hill, ON, Canada), but this procedure 
could easily be automated within the CIS operations. The SAR images supplied by CIS are all eight-bit coded, block-averaged to $100 \mathrm{~m}$, and registered to a Lambert conical conform projection. The SAR images are transformed in an eight-bit standard BIL format from which the texture features are calculated using the PCI Modeler texture generation routine.

Three GLCM statistics are recommended for classification of pure sea ice sample [5], [28]: contrast, entropy, and correlation. However, for segmentation purposes, windows often have mixed class types, and the correlation statistic has been demonstrated to generate erroneous class boundaries [31]. As a result, here only contrast and entropy are used. Tests (not shown here) have led to the selection of a window size of $9 \times 9$ pixels and a single pixel pair distance of two. A quantization level of 64 gray level is used [28], and the GLCM statistics were averaged over four directions $\left(0^{\circ}, 45^{\circ}, 90^{\circ}\right.$, and $\left.135^{\circ}\right)$ to generate rotationally invariant features.

\section{B. Components of the System}

The system is composed of five modules (or programs) that are sequentially arranged and that could eventually be merged into a single program (Fig. 2). They have been developed separately because they correspond to different conceptual operations, and this also makes the description of the system conceptually simpler. The starting point for the system is provided by the three data sources described above; namely a registered SAR image and egg code map pair as well as an attribute file accompanying the egg code map containing for each egg region the corresponding ice/open water percentage along with the ice types and percentage. The five components of the system are described below.

1) SCANEGG: The first program reads the attribute file (in a spreadsheet format) and the egg code map. SCANEGG then determines for each egg code region the minimal enclosing rectangular subimage coordinates and writes these coordinates (along with the ice and open water data) in an output file so that the amount of memory needed by the segmentation routine, when applied to the circumscribing rectangles, is minimized. This precaution is especially important with the use of texture features to complement the backscattering values of the SAR image in the segmentation process. The MRF routine needs to keep the whole region and features in memory making this precaution necessary to minimize completion time.

2) MAp-Guided Sea-Ice Segmentation: The "MAp-Guided Sea-Ice Segmentation" (MAGSIS) is the second component and the most time consuming. Each egg code region (defined by its minimal enclosing subimage) is stored in memory along with the corresponding SAR subimage and texture features and sent to the MAMSEG, which is fully described in [2] and briefly explained in the next paragraph. The MRF segmentation routine returns a segmented image that is inserted into the corresponding sea ice map. MAMSEG needs to know the number of classes as supplied by the egg code information. No more than three sea ice classes are normally included in a single egg code. If open water is present (i.e., the overall ice concentration is $90 \%$ or less), an additional class is considered. Fig. 1 shows an egg code example with three ice type classes and open water (10\%). For example, for a three-sea-ice-classes egg code region with open water, the MAMSEG routine will return an image containing labels between one and four but will not provide any information as to which label represents which class. The problem of assigning the proper label to each segment for all egg code regions is defined here as the "labeling problem."

3) MAMSEG: This subcomponent is the core subsystem that actually performs the segmentation work based on the MRF model. Full details of this model are found in [2]. The model is based on the Bayesian framework formulated as

$$
P\left(G_{i} \mid x\right)=\frac{p\left(x \mid G_{i}\right) P\left(G_{i}\right)}{\sum_{i}\left[p\left(x \mid G_{i}\right) P\left(G_{i}\right)\right]}
$$

where $p\left(x \mid G_{i}\right)$ is the conditional distribution for feature vector $x$ given class $G_{i}$, and $P\left(G_{i}\right)$ is the a priori probability for class $G_{i}$. Suppose the energy associated with the a priori probability is $E_{r}$ and that $E_{f}$ represents the energy form of $p\left(x \mid G_{i}\right)$. Then the general energy form for $P\left(G_{i} \mid x\right)$ is given by [37]

$$
E=E_{r}+\alpha E_{f}
$$

where $\alpha$ is a weighting parameter used to determine the respective proportions of $E_{r}$ and $E_{f}$ 's individual contribution to $E$. Whereas MRF models traditionally assume a fixed value for $\alpha$, the modified version [2] decreases $\alpha$ for each iteration in the simulated annealing solution that seeks to minimize $E$. This ensures that $E_{f}$ first dominates the MRF model ( $\alpha$ is large) in order to learn its global mean $(\mu)$ and standard deviation $(\sigma)$ and yields more importance to the local label as the model shifts toward a solution with each iteration.

Normally, MRF models are initialized with a random image segmentation, which has been demonstrated to work well in most situations [2], [32]. However, in this implementation, by providing an initial segmentation based on the backscattering intensity and using the K-means method, the number of iterations in the MRF procedure is typically reduced by 10 to 20 while maintaining similar results. For the examples presented here and in other similar tests, 50 iterations were generally sufficient.

4) Map-Guided Sea-Ice Statistics: "Map-guided sea-ice statistics" (MAGSISTAT) calculates the mean and the covariance matrix $(\mu$ and $\Sigma$ ) of each and among the features (backscattering and texture) for each segment (or segmented class) of each egg code region. It also computes and stores the histogram for each feature. MAGSIS returns integer labels but makes no inference as to which label represents which class. MAGSISTAT builds a spreadsheet where each segment of each egg code region is a row entry. For each row entry, the $\mu$ and $\Sigma$ of each feature are calculated, and all possible classes are also recorded. To illustrate this process, a spreadsheet example is shown in Table I (covariance matrices and histograms are stored in the same spreadsheet but are not shown here) where a single egg code region having a value of 46 (corresponding to "A" in Fig. 1) in the egg code map was segmented into four separate classes having pixel values between one and four (in the ice map) which correspond to one of the following classes: 7 (thin first-year ice), 5 (gray-white ice), 4 (gray ice), or 20 (open water). Note that "open water" is not part of the WMO ice coding, so, for the purpose of this implementation, the code " 20 " has been assigned. The analyst-estimated percentage of each ice type is also recorded but not used at this stage because 
TABLE I

EXAMPLE OF A SPREADSHEET RETURNED BY THE MAGSISTAT PROGRAM. ECR Is THE EGG CODE REgION VALUE. L IS THE LABEL. $n_{c}$ Is THE NUMBER of Classes. C1-4 Are the Four Possible ICE Classes. \%C1-4 ARE THE CONCENTRATIONS FOR EACH ICE ClASS. $\overline{F_{i}}$ ARE THE MEANS of EACH FEATURE (SAR INTENSITIES ARE RESAMPLED TO EIGHT Bits, TeXture FeATURES to 16 BITS); FL Is THE ICE LABEL EACH SEgment RECEIVED AFTER Classification

\begin{tabular}{rrrrrrrrrrrrrrrr}
\hline ECR & $\mathrm{L}$ & $n_{c}$ & $\mathrm{C} 1$ & $\mathrm{C} 2$ & $\mathrm{C} 3$ & $\mathrm{C} 4$ & $\% \mathrm{C} 1$ & $\% \mathrm{C} 2$ & $\% \mathrm{C} 3$ & $\% \mathrm{C} 4$ & $\overline{F_{1}}$ & $\overline{F_{2}}$ & $\overline{F_{3}}$ & $\mathrm{FL}$ \\
\hline 46 & 1 & 4 & 7 & 5 & 4 & 20 & 30 & 30 & 30 & 10 & 46.11 & 36378.6 & 22613.4 & 0 \\
46 & 2 & 4 & 7 & 5 & 4 & 20 & 30 & 30 & 30 & 10 & 108.0 & 47051.8 & 30837.6 & 0 \\
46 & 3 & 4 & 7 & 5 & 4 & 20 & 30 & 30 & 30 & 10 & 95.1 & 33971.3 & 34585.5 & 0 \\
46 & 4 & 4 & 7 & 5 & 4 & 20 & 30 & 30 & 30 & 10 & 168.6 & 39402.3 & 28707.2 & 0 \\
\hline
\end{tabular}

the margin of error is too large (this has been confirmed by CIS personnel) for the program to consistently use as a prior probability. Note that the last column named "final ice type label" received an initial label of zero for all entries. This indicates that the classification program (described below) has not yet solved the class label for this segmentation result. As an additional precaution, the MAGSISTAT program excludes from the calculation all pixels fewer than four pixels away from an egg code region edge. This is performed by applying a Laplacian kernel of $7 \times 7$ to the segmented map and excluding all output pixels for which the result differs from zero. This precaution was taken to account for the approximate manner by which the analysts draw their egg code polygons.

5) Sea-Ice Classification by Cognitive Reasoning: The "sea-ice classification by cognitive reasoning" (SICLASSCORE) program tries to perform the classification (which has been reduced to an association problem since the number of classes and the categories are already known) by accumulating evidences and "learning" solutions as it proceeds (see example at the end of this section). Three types of evidence are defined: first, second, and third degree. First-degree evidence falls into two categories: 1) the egg code region contains only one class or 2) the egg code region contains several classes, and all but one have already been solved and assigned. In either case, the association is straightforward, and no additional information is needed to solve the association. The $\mu$ and $\Sigma$ values for the "solved" segment are then retained and associated to the final class. Each time a new "solution" is found, the program calls a subroutine that automatically updates the statistics of the class (see below).

Second-degree evidence is characterized by the fact that although all or some classes of an egg code region have previously been solved (in other egg code regions), the program still has to find which set of associations is the most likely. For a total of $n_{c}$ classes, there are $n_{c}$ ! permutations of matching each segment of a particular egg code region to one of the $n_{c}$ classes. The objective is then to determine which permutation is more likely according to some metric. Three methodologies for performing the optimal label assignment are presented in Section III-C: Fisher (nonparametric), Mahalanobis (distance), and Kolmogorov-Smirnov (probabilistic). Any of these methods could be used, and for the current discussion the term "metric" is used to describe any one of these. The means $(\mu)$, covariance matrices $(\Sigma)$ and histograms $(H)$ of the solved classes are kept in a series of $m_{c} \times m_{f}$ arrays where $m_{c}$ represents the number of classes and $m_{f}$ the number of features.
These arrays have a third dimension to store the values of the $\Sigma$ 's and H's.

The association set with optimal cumulated metric is retained as the most likely solution. Again, the class statistics are updated after each new solution is found. The program starts by solving egg code regions with two classes where all classes have been solved previously and then does the same for egg code regions with three and four classes. Then the program solves cases of two known classes in three-class regions and three known classes in four-class regions and so on.

In third-degree evidence, reasoning is based on the fact that while comparing two egg code regions, although no association was previously solved, if only one class is common to both egg code regions (intersection), then one can deduce which is more likely by calculating a distance metric between all pairwise possibilities. The optimal result is retained as the correct association.

Each time a segment is found to belong to a particular class, regardless of the degree of evidence, that class can be updated with the new segment population. Hence, for the segment $i$ having $n_{i}$ samples (or pixels) and found to belong to class $j$ (with $n_{j}$ samples), the covariance matrices are merged using the following closed-form update equation [43, p. 119]:

$$
\Sigma_{i j}=\frac{S_{i j}}{\left(n_{i}+n_{j}\right)}
$$

where

$$
S_{i j}=S_{i}+S_{j}+\left(\mu_{i}-\mu_{j}\right) \cdot\left(\mu_{i}-\mu_{j}\right)^{t} \frac{n_{i} n_{j}}{n_{i}+n_{j}}
$$

is the result of merging the scatter matrices of segments $i$ and $j$ defined by $\Sigma_{i} n_{i}$ and $\Sigma_{j} n_{j}$, respectively. Similarly, the means are updated using the relation

$$
\mu_{i j}=\frac{\mu_{i} n_{i}+\mu_{j} n_{j}}{n_{i}+n_{j}} .
$$

6) Sea-Ice Reclassification: Having gathered all the evidence and stored them in a lookup table, "sea-ice reclassification" (SIRECLASS) performs the last step by reclassifying (assigning the final label to each segment) the sea ice map segments by cross tabulation. Using the example in Table II, initial label 1 of egg code region 1 acquires ice type "1"; initial label 2 of the same egg code region becomes " 4 " and so on for the other regions.

\section{Metrics Used for Label Assignment}

Three different methods were considered in SICLASSCORE to calculate the likeliness of a segment belonging to a class: 1) the Fisher criterion (FC) [43, p. 117]; 2) the Mahanalobis distance (MD) [43, p. 36]; and 3) the Kolmogorov-Smirnov (KS) $[44$, p. 623] test. The first method is based on the Fisher criterion $J(i, j)$

$$
J(i, j)=\frac{\omega_{i j}^{t} S_{B} \omega_{i j}}{\omega_{i j}^{t} S_{W} \omega_{i j}}
$$

where $S_{B}=\left(\mu_{i}-\mu_{j}\right)\left(\mu_{i}-\mu_{j}\right)^{T}$ and $S_{W}=S_{i}+S_{j}$ are the between- and pooled within-class scatter matrices, respec- 
TABLE II

EXAMPLE OF A SPREADSHEET RETURNED BY THE MAGSISTAT PROGRAM. LEGEND: 1-NEW ICE; 4-GRAY ICE; 5-GRAY-WHITE ICE; 7-THIN FIRST-YEAR ICE; 1. MEDIUM FIRST-YEAR ICE; 20-OPEN WATER. ECR IS THE EGG CODE Region VAlue. L Is the LABEL. $n_{c}$ Is THE NuMber OF Classes. C1-4 ARE THE Four Possible IcE Classes. FL Is THE ICE LABEL EACH SEGMENT RECEIVEd AFTER ClassifiCATION. NOTE THAT THE ORIGINAL WMO CODES From THE EGG CODED MAP WERE KEPT WHERE A TO Z REPRESENT ICE-INFESTED POLYGONS AND OW REPRESENTS AN OPEN WATER REGION. SOME OF THE EGG CODE REgIONS HAVE BEEN LEFT OUT FOR SIMPLIFICATION

\begin{tabular}{rrrrrrrr}
\hline $\mathrm{ECR}$ & $\mathrm{L}$ & $n_{c}$ & $\mathrm{C} 1$ & $\mathrm{C} 2$ & $\mathrm{C} 3$ & $\mathrm{C} 4$ & $\mathrm{FL}$ \\
\hline $\mathrm{OW}$ & 1 & 1 & 20 & 0 & 0 & 0 & 20 \\
\hline $\mathrm{A}$ & 1 & 2 & 4 & 1 & 0 & 0 & 1 \\
$\mathrm{~A}$ & 2 & 2 & 4 & 1 & 0 & 0 & 4 \\
\hline $\mathrm{C}$ & 1 & 2 & 1 & 7 & 0 & 0 & 7 \\
$\mathrm{C}$ & 2 & 2 & 1 & 7 & 0 & 0 & 1 \\
\hline $\mathrm{J}$ & 1 & 3 & 7 & 5 & 4 & 0 & 7 \\
$\mathrm{~J}$ & 2 & 3 & 7 & 5 & 4 & 0 & 4 \\
$\mathrm{~J}$ & 3 & 3 & 7 & 5 & 4 & 0 & 5 \\
\hline $\mathrm{W}$ & 1 & 3 & 7 & 5 & 20 & 0 & 5 \\
$\mathrm{~W}$ & 2 & 3 & 7 & 5 & 20 & 0 & 20 \\
$\mathrm{~W}$ & 3 & 3 & 7 & 5 & 20 & 0 & 7 \\
\hline $\mathrm{N}$ & 1 & 3 & 7 & 5 & 20 & 0 & 5 \\
$\mathrm{~N}$ & 2 & 3 & 7 & 5 & 20 & 0 & 20 \\
$\mathrm{~N}$ & 3 & 3 & 7 & 5 & 20 & 0 & 7 \\
\hline $\mathrm{M}$ & 1 & 3 & 7 & 5 & 4 & 0 & 7 \\
$\mathrm{M}$ & 2 & 3 & 7 & 5 & 4 & 0 & 4 \\
$\mathrm{M}$ & 3 & 3 & 7 & 5 & 4 & 0 & 5 \\
\hline $\mathrm{Z}$ & 1 & 3 & 1. & 7 & 5 & 0 & 1. \\
$\mathrm{Z}$ & 2 & 3 & 1. & 7 & 5 & 0 & 5 \\
$\mathrm{Z}$ & 3 & 3 & 1. & 7 & 5 & 0 & 7 \\
\hline $\mathrm{O}$ & 1 & 4 & 7 & 5 & 4 & 20 & 7 \\
$\mathrm{O}$ & 2 & 4 & 7 & 5 & 4 & 20 & 20 \\
$\mathrm{O}$ & 3 & 4 & 7 & 5 & 4 & 20 & 5 \\
$\mathrm{O}$ & 4 & 4 & 7 & 5 & 4 & 20 & 4 \\
\hline $\mathrm{V}$ & 1 & 4 & 1. & 7 & 5 & 20 & 1. \\
$\mathrm{~V}$ & 2 & 4 & 1. & 7 & 5 & 20 & 5 \\
$\mathrm{~V}$ & 3 & 4 & 1. & 7 & 5 & 20 & 20 \\
$\mathrm{~V}$ & 4 & 4 & 1. & 7 & 5 & 20 & 7 \\
\hline & & & & & & &
\end{tabular}

tively, and $\omega_{i j}=\Sigma_{i j}^{-1}\left(\mu_{i}-\mu_{j}\right)$. A Fisher criterion is calculated for each class pair, and the segment is assigned to the class with the smallest $J$. The Fisher criterion offers the advantage of taking into consideration the spread of both distributions being compared.

The Mahanalobis distance is a very popular classification method in the remote sensing community. It is equivalent to the maximum-likelihood classifier when all a priori probabilities are equal

$$
r^{2}(\mathbf{x}, i)=\left(\mathbf{x}-\mu_{i}\right)^{t} \Sigma_{i}^{-1}\left(\mathbf{x}-\mu_{i}\right) .
$$

A Mahanalobis distance is normally calculated between a sample $\mathbf{x}$ (pixel vector) and a class distribution $\left(\mu_{i}, \Sigma_{i}\right)$; therefore, it does not take into account the spread when a bundle (a whole segment) is considered instead of a single observation.

Finally, the KS test computes the probability of two distributions belonging to the same population based on the distance between their cumulative distributions

$$
D(i, j)=\max _{-\infty<x<\infty}\left|C H_{i}(x)-C H_{j}(x)\right|
$$

where $\mathrm{CH}_{i}(x)$ and $\mathrm{CH}_{j}(x)$ represent the frequency of occurrence of bin (pixel value) $x$ in the two cumulative frequency distributions. The probability that $D(i, j)$ is significant (reject the null hypothesis that the two distributions are the same) is then computed using the following sum:

$$
Q_{\mathrm{KS}}(\lambda)=2 \sum_{i=1}^{\infty}(-1)^{i-1} e^{-2 i^{2} \lambda^{2}}
$$

where $\lambda=D(i, j)\left(\sqrt{N_{e}}+0.12+0.11 / \sqrt{N_{e}}\right)$ and $N_{e}=$ $\left(\left(N_{1} N_{2}\right) /\left(N_{1}+N_{2}\right)\right)$. Thus, the larger $Q_{\mathrm{KS}}$, the less likely the two distributions belong to the same population.

With all of these methods, the metrics are cumulated for each combination of assignments, and either the smallest (MD or FC) or largest (KS probability) value is retained as the correct solution.

\section{Example}

To illustrate the complete process, the spreadsheet of part of an egg code map with ten egg code regions is shown in Table II. The following paragraphs explain how the evidence is gathered and the labels attributed.

- The program first finds evidence that the egg code region OW (open water) has only one class (first-degree evidence): class 20 is solved.

- Then it checks if one of the two-class egg code regions also has class 20 in which case it could figure out which is class 20 and which is the remaining class (second-degree evidence).

- Since this is not the case, it looks for third-degree evidence: no class has been solved but there is a unique class common to two "two-class" egg code regions: this is the case between egg code regions $\mathrm{A}$ and $\mathrm{C}$ where class 1 is uniquely shared. It is found that the first class of egg code region $\mathrm{A}$ and the second class of egg code region $\mathrm{C}$ correspond to class 1: 1 and 20 are solved.

- Then, since one class of both egg code regions A and C is known, first-degree evidence tells us that the remaining class of each egg code can only belong to the other possible class: class 4 for egg code region A and class 7 for egg code region $\mathrm{C}: 4,7,1$, and 20 are solved.

- Although four classes have been solved there are no remaining egg code regions having all its possible classes previously solved but three of the four remaining "threeclass" egg code regions have two out of three classes solved and so second-degree evidence can be used to determine which segments are more likely to belong to these classes.

- First-degree evidence can now be used to solve class 5 in region W. Now, 5, 4, 7, 1, and 20 are solved.

- Again, second-degree evidence can be used to find which of the three classes of region $\mathrm{Z}$ is class 7 and 5 leaving only class 1 (one dot, a WMO code) as the only class remaining unsolved.

- First-degree evidence is used to solve class 1 in Z. Now, 1., $5,4,7,1$, and 20 are solved.

- Finally, second-degree evidence can be applied to find the most likely combination for the last two egg code regions 


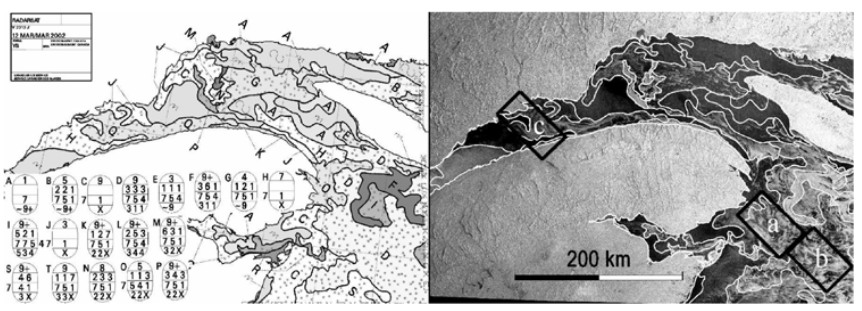

(a)

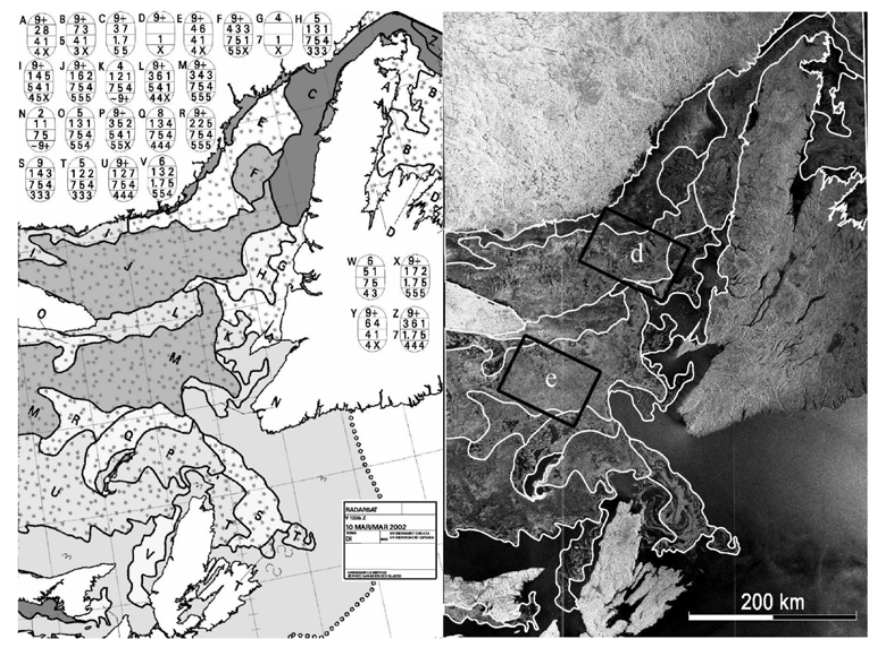

(b)

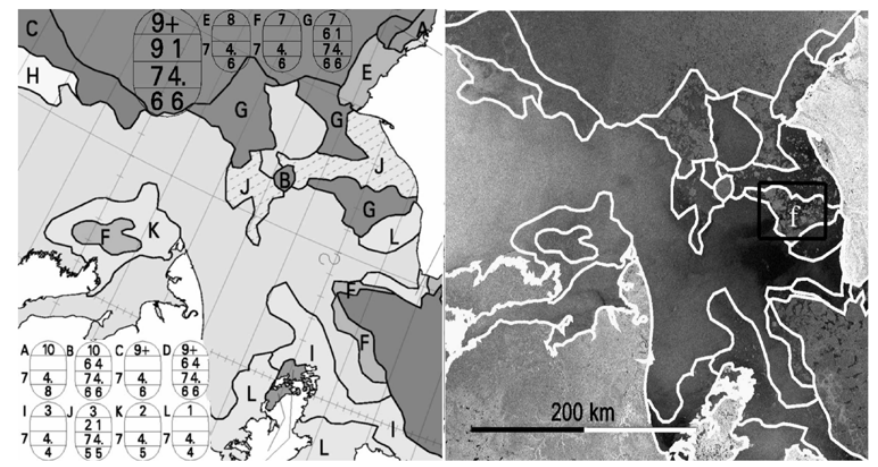

(c)

Fig. 3. (Left) Egg code maps and (right) Radarsat images for the three examples. (a) Western (March 10, 2002) and (b) Eastern (March 13, 2002) Gulf of Saint Lawrence and (c) the Beaufort Sea (October 13, 1997). Marked areas represent the locations of the samples of Fig. 4.

having four classes (regions $\mathrm{O}$ and $\mathrm{V}$ ) which have all been previously solved.

- If not all cases have been solved, the process can be continued by testing weaker evidence (e.g., only one class is known in three or four).

Even considering that typical egg code maps will provide sufficient evidence for solving all classes of all egg code regions, there still exists, in theory, the possibility that no strong evidence is given as a starting point. In these cases we are considering "helping" the program by providing training data to be used as a starting point for one or two classes; this should provide enough first-degree evidence to trigger the reasoning for solving the remaining classes.

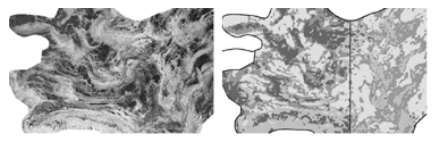

(a)

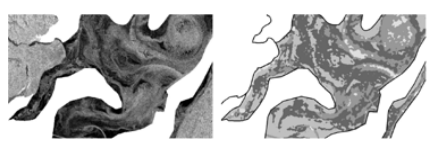

(c)

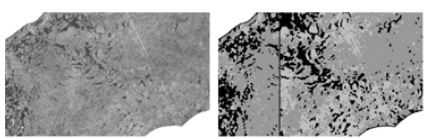

(e)
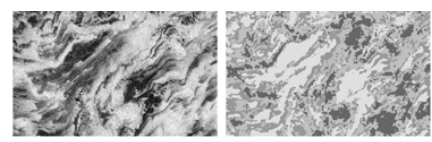

(b)
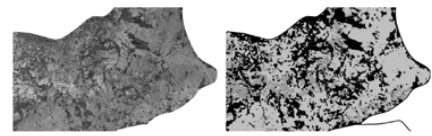

(d)
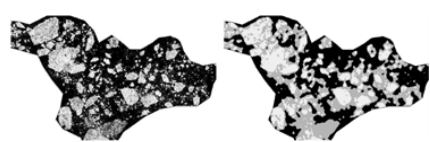

(f)
Fig. 4. Original SAR image samples (left side of subfigures) and segmentation result (right side). (a)-(c) Eastern Gulf of Saint Lawrence SAR image. (d)-(e) Western Gulf. (f) Beaufort Sea image. Note that (a) and (e) had to be segmented in two stages. The black vertical lines show where the split was performed. Location of each sample is shown in Fig. 3. All images are compressed in size for display purposes and have 430 lines of 700 pixels (approximately $70 \mathrm{~km}$ in width and $43 \mathrm{~km}$ in height).

\section{RESUlTS AND DISCUSSION}

Results are presented for three Radarsat-1 scan mode images: two of the Gulf of Saint Lawrence, one for the eastern part (March 13, 2002) and one in the western end (March 10, 2002) and one of the Beaufort Sea region (Fig. 3). One image is the result of merging two swaths causing a straight vertical linear feature that crosses the entire image scene [can be seen crossing region E in Fig. 3(b)]. These images results from direct CIS operations and have not received any alteration or cropping (except for display purposes) to try to simulate a real operational context. The first two images are also considered "difficult cases" according to CIS (D. Flett, personal communication, October 2004). Reporting realistic success and error in the segmentation and classification of sea ice is a rather difficult task since ground truth is very difficult to obtain due to the highly dynamic and inaccessible nature of sea ice. On the other hand, since only one element is considered in its various states (from open water to multiyear ice), and SAR images are the main source of data, the interpretation is almost solely a visual task. It is only natural then to evaluate the performance of segmentation on a visual basis rather than only relying on often too few control samples. The evaluation was also done by consulting an ice analyst from CIS. Three levels of evaluation are considered here: 1) segmentation; 2) classification; and 3) operational.

\section{A. Segmentation}

Fig. 4 shows a series of six segmentation examples drawn from the three SAR images. The samples were selected for having at least three classes (the maximum being four) and for illustrating different patterns of sea ice where texture played an evident role. Two of these examples [Fig. 4(a) and (e)] were split in two parts which were segmented separately (region splitting was sometimes necessary to reduce the amount of memory used). In both cases (and in all other cases not shown here) the segmentation was consistent across these artificial 
borders. Segmentation results were rated based on two separate evaluations. The first evaluation aimed at finding the ability of the segmentation to preserve natural edges and small details (Table III). Results were rated negatively when artifact boundaries were created and when borders with other egg code regions were inconsistent. The rating criteria were as follows. "All or Most" is attributed when the segmentation appears to have captured all or most important features with a good level of detail. "Partial" implies that some important feature patterns are not properly rendered. "Few" indicates that many important feature are absent. Table III shows that the segmentation captured most visual details for the great majority (55/78) of the egg code regions for all SAR images. In all 18 egg code regions were rated as "Partial" and five as "Few." It should be noted that many egg code regions had inconsistencies such as a missing an ice class or that the egg code region contour was not precisely drawn and partially overlayed a neighboring class not present in the egg code.

The addition of texture helped to preserve the small details since these are characterized by good local contrast. However, since texture features are window-based, edges tend to be exaggerated in size and so are the small features. Although this is not desirable, it still is better than the opposite situation that would involve the disappearance of these details. Additionally, the amount of exaggeration of these details is related to the number of iterations and the number of classes in the MRF segmentation routine. More iterations result in these small details to be either exaggerated in size or to disappear when contrast is low. Likewise fewer classes tend to have the same effect. One possible way to counter this problem would be to use the number of classes as a basis for calculating the number of iterations necessary. Additional testing needs to be performed to model this effect more precisely.

The second evaluation involved an ice analyst from CIS to characterize the match between the segments and the actual ice types within the egg code regions. Ice analysts are professionals (usually from a physics of meteorology background) whose work consists in analyzing and interpreting data from various sources (one of which is usually a SAR image) to produce daily ice charts. This validation made it possible to identify not only the accuracy of the segmentation but mostly, it has outlined the different problems that the segmentation was facing. Upon confronting the results with ice analysts, it became quite clear that many discrepancies between the analyst's interpretation and the computer-generated segmentation could be attributed to four principal factors:

1) Incomplete information in the egg codes. Space limitations can force the analyst to sometimes ignore a class (usually the one with the smallest percentage or the thinnest ice). The analyst also knows that their interpretation will be later generalized (in a broader region), and some similar egg codes might be merged with others disregarding the least important ice classes. Imprecise delineation of the egg code region polygons can also incorporate classes for which the egg code has no information (some egg code regions have incorporated parts of neighboring regions or even land or fast ice).
TABLE III

SEGMENTATION Visual RATINGS FOR THE EGG CODE REGION FOR THE THREE SAR IMAGES: $E=$ EASTERN GUlF OF SAINT LAWRENCE,

$W=$ Western Gulf of SaINT LaWrence, $B=$ Beaufort SEA. THE FIGURES REPRESENT THE NUMBER OF EGG CODE REGIONS

\begin{tabular}{rrrr}
\hline SAR image & All or Most & Partial & Few \\
\hline E (13 March 2000) & 21 & 6 & 3 \\
W (10 March 2002) & 22 & 5 & 0 \\
B (7 July 2004) & 12 & 7 & 2 \\
\hline Total & 55 & 18 & 5 \\
\hline
\end{tabular}

2) Ancillary information. The analyst has access to information from neighboring regions, previous days, and from other sources (space, airborne, or ground-based). This explains for instance how ice floe sizes smaller than the image resolution can sometimes be indicated in the egg code.

3) Shape and size. Some ice classes are directly related to the shape and size of ice floes. For instance, first-year ice can often be separated from gray-white ice because the former is characterized by older floes that appear to have smoother boundaries due to constant grinding contact with other floes. This cue is not yet taken into account as a separate feature.

4) Angle of incidence. The angle of incidence can have a dramatic effect on the backscattering (especially of open water in windy conditions) and this has sometimes caused a single class to be split in two distinct segments at the expense of another class with subtle differences.

Table IV is a synthesis of the validation process in which these four problems were organized according to the origin of the flaw as follows:

1) Incomplete information

a) missing information in egg code;

b) interpretation was based on ancillary data;

\section{2) Poor performance of the segmentation}

a) segmentation should incorporate information on shape and size;

b) incidence angle problems (shift in brightness)

The ice analyst's validation revealed that 43 of the 78 egg code regions suffered from at least one of the four problems. Incomplete information (1a and 1b) affects at least 28 egg code regions (36\%) and poor performance of the segmentation algorithm ( $2 \mathrm{a}$ and $2 \mathrm{~b}$ ) due to missing features (shape and size) or incidence angle problem was detected in at least 30 regions $(38 \%)$. While the latter problems could potentially be taken into account by the segmentation algorithm, the former cannot be solved at this level. Other reasons might be responsible for unsatisfactory segmentation that do not fall in one of these categories but these appear to be in a very small proportion.

It should also be mentioned that while doing their interpretation, the ice analyst does not try to identify the ice classes individually but rather tries to grasp the situation in a much more dynamic way. The analysts visualize movement and progression, which are important cues in their final decision. On the other hand, the computer program in its actual form tries to identify these classes independently of their context in a static approach. 
TABLE IV

VALIDATION OF THE SEGMENTATION RESULTS FOR THE THREE SAR IMAGES. $E=$ EASTERN GULF OF SAINT LAWRENCE, $W=$ WESTERN GULF OF SAINT LAWrence, B = BEAUfort Sea. THE Figures RePRESENT THE Number of Egg Code Regions. Note That a Single EgG Code Region CAN SufFer From More Than ONe Problem

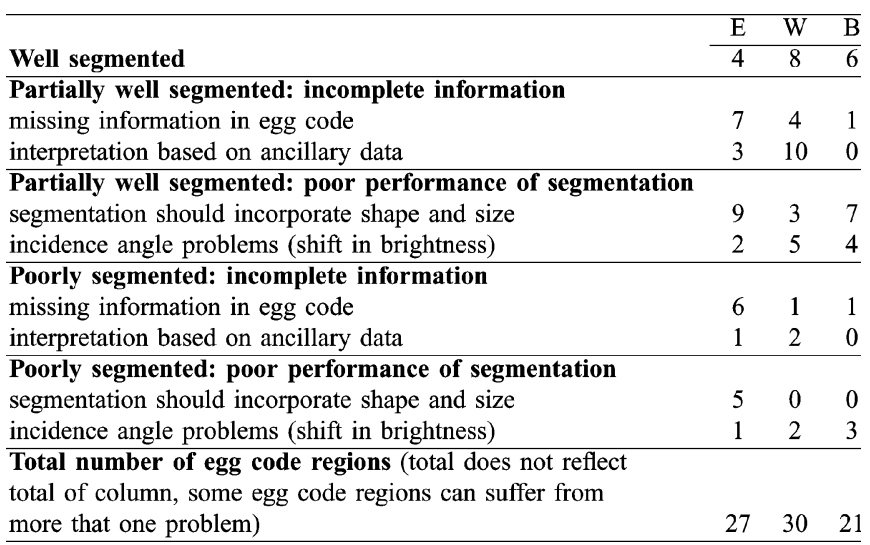

These are two very different levels of description. Hofstadter describes one of the challenge of artificial intelligence (and computer vision by extension) as "bridging the gap between these two descriptions: how to construct a system which can accept one level of description and produce the other" [45, p. 285]. He makes an analogy with the comparison of how novices and masters perceive a chess situation: while the novices see the position of individual pieces on the chess board, the masters are sensitive to high-level patterns or types of recurring situations-they see the chess board on a higher level. In the case of sea ice interpretation, the computer program is the novice who does not yet see the greater picture. It will need not only more features but also more ways of combining them.

The analysis of the results by ice analysts was very rewarding in that it pin-pointed not only the problems but also many of the possible solutions. For instance, it appears that the egg codes should be first analyzed on a high level to identify cases where information might be missing or be more detailed than what the segmentation can be expected to achieve. For example, in cases where the ice floes are smaller than the resolution of the image, some distinctions between ice types are not possible without additional airborne or ground information.

\section{B. Classification}

As mentioned above, reporting classification success without ground truth is not absolute, but is recognized to be an acceptable procedure given the particularities of sea ice mapping. The labeling was validated using the ice analysts experience to check the appropriateness of the classification given the limitations of the segmentation. Egg code regions that were properly segmented were used directly and the number of correctly labeled segments were simply counted. Egg code regions that suffered from poor segmentation could not be used directly so the number of correctly labeled segments were counted against the number of correctly segmented classes. For example, if an egg code region had four classes but only two were appropriately segmented, then only these two segments'
TABLE V

CLASSIFICATION RATINGS REPRESENTING THE NUMBER OF EGG CODE REGIONS CORRESPONDING TO EACH CRITERION FOR THE THREE CLASSIFIERS AND THE THREE SAR IMAGES IN FIG. 5 (E FOR EASTERN GUlF OF SAINT LAWRENCE, W FOR WESTERN, AND B FOR BEAUfORT SEA)

\begin{tabular}{|c|c|c|c|c|c|c|c|c|c|c|c|c|}
\hline Method used & & isher & titerio & & & $\begin{array}{l}\text { Maha } \\
\text { Distanc }\end{array}$ & $\begin{array}{l}\text { alobis } \\
\text { (MD }\end{array}$ & & & $\begin{array}{l}\text { Kolmo } \\
\text { Smirno }\end{array}$ & $\begin{array}{l}\text { gorov- } \\
\text { (KS) }\end{array}$ & \\
\hline SAR image & E & $\mathrm{W}$ & B & $\bar{x}$ & E & W & B & $\bar{x}$ & E & W & B & $\bar{x}$ \\
\hline Completely usable & & & & & & & & & & & & \\
\hline egg code classes & $\frac{15}{17}$ & $\frac{4}{6}$ & $\frac{12}{12}$ & & $\frac{17}{17}$ & $\frac{4}{6}$ & $\frac{12}{12}$ & & $\frac{11}{17}$ & $\frac{6}{6}$ & $\frac{6}{12}$ & \\
\hline Percentage (\%) & 88.2 & 66.7 & 100 & 85.0 & 100 & 66.7 & 100 & 88.9 & 64.7 & 100 & 50 & 71.6 \\
\hline Partially usable & & & & & & & & & & & & \\
\hline egg code classes & $\frac{25}{35}$ & $\frac{20}{31}$ & $\frac{15}{22}$ & & $\frac{30}{35}$ & $\frac{18}{31}$ & $\frac{15}{22}$ & & $\frac{20}{35}$ & $\frac{11}{31}$ & $\frac{13}{22}$ & \\
\hline Percentage (\%) & 71.4 & 64.5 & 68.2 & 68.0 & 85.7 & 58.1 & 68.2 & 70.7 & 57.1 & 35.5 & 59.1 & 50.6 \\
\hline
\end{tabular}

labels were considered. This is not trivial because the number of possibilities remains the same so the fact that one class is correct does not imply that the second is also correct. In some cases, the segmentation might have missed part of a class but the labeling could still assign the most appropriate label to that segment enabling that egg code region to be considered for validation.

Table V shows the ratings for the three images separately. The table is divided according to whether the classes of the egg code were all (second row) or partially usable (third row). This criterion was based on the evaluation of the segmentation as there was no point in validating a class that was not well segmented to start with. The top value of each row represents (as a fraction) the proportion of accurately classified classes on the total number of usable classes and the bottom value gives the corresponding percentage. Given about $89 \%$ of classification accuracy for well-segmented regions and $71 \%$ for partially well segmented regions, the MD metric outperformed the other two methods, but the difference with FC is small and might not be significant. The poorer performance of the KS metric (only $36 \%$ egg code regions with no apparent errors) can probably be attributed to the fact that it is too sensitive to the inaccuracies associated with not knowing the true class distributions (e.g., histograms having skewed shape and suffering from shifts in central tendency (mean, mode or median) due to varying angle of incidence). This is especially true for the Beaufort image for which this effect was strong in the open water. In general, open water has a dark SAR intensity and a rather smooth texture and this is the case for the majority of regions in both test images. However, the presence of waves (wind) coupled with the varying incident angle can produce extreme differences in both the intensity and the texture of open water [15], [46].

One obvious limitation of the classification is that it can only attribute a label that already exists in the egg code so that any imprecise contouring from the ice analyst can include an unaccounted class in the egg code region that would probably affect the segmentation algorithm and the classification consequently. A possible solution to this would be to perform an oversegmentation and to consider a "mystery" class during the initial labeling process. A subsequent labeling would associate the "mystery" segment to the most probable class from the set of classes from the whole image (and not only an egg code region). This technique is currently being considered for future versions of the system. 


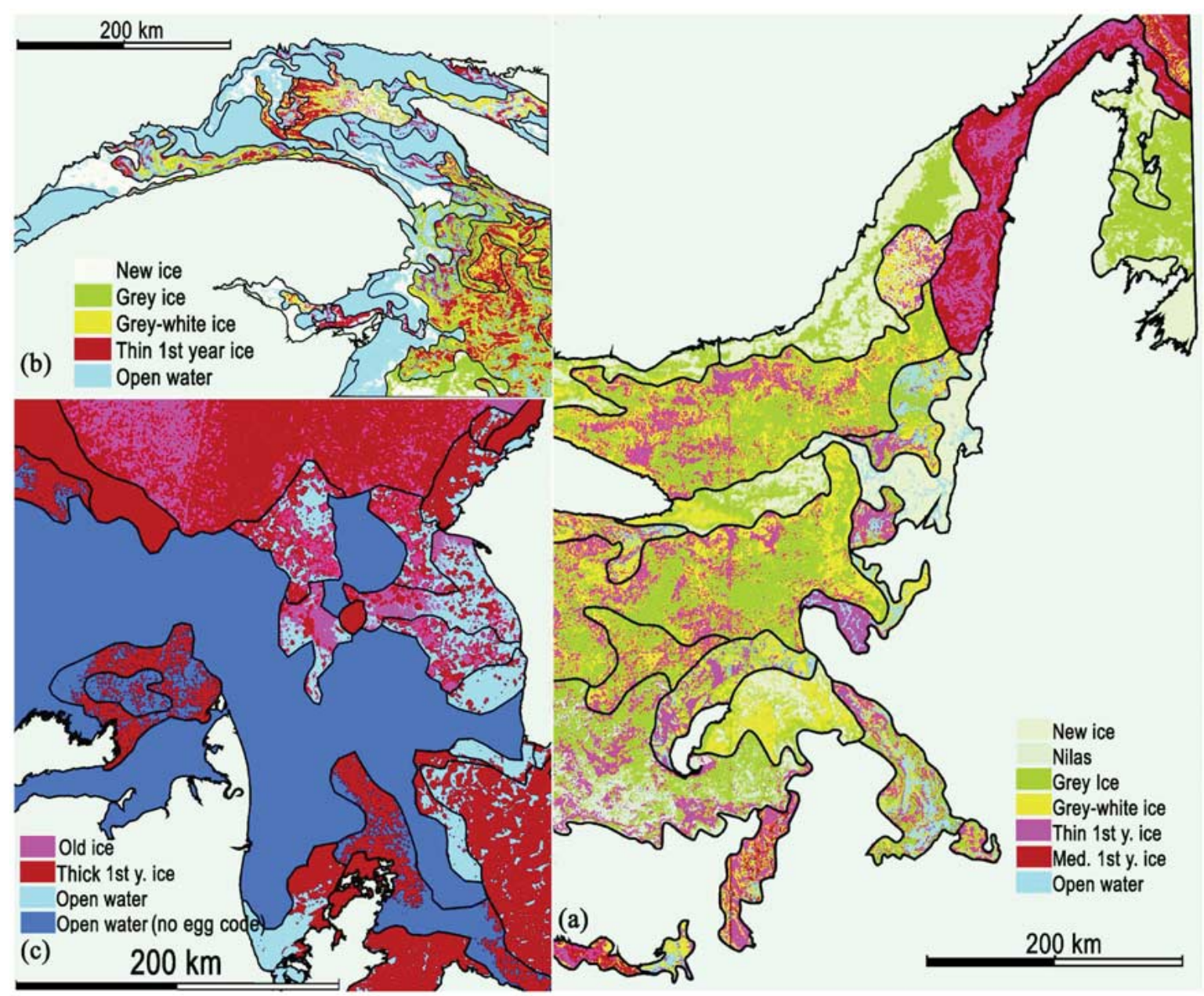

Fig. 5. Classification results for the (a) Eastern (March 13, 2002) and (b) Western (March 10, 2002) Gulf of Saint Lawrence and (c) the Beaufort Sea (October 13, 1997).

Nevertheless, the classification results are correct by an appreciable majority for the MD classifier. Upon analyzing the errors, it was found that many cases are benign and involve classes that are alike with similar impact such as gray and gray-white ice or medium- and thin-first year ice. However, there are a few errors involving confusion between open water and ice which have more dramatic consequences. Another factor that caused such confusion is the apparent likeliness of some ice categories with open water. For instance, some egg code regions do not include new ice but have features that resemble it strongly. It is also possible that the egg code region contains some new ice but that the ice analyst did not judge it worthwhile having mainly navigation in mind (D. Flett, personal communication, October 2004). Several approaches have been contemplated to solve this problem but were not implemented at this stage. This particular topic (separation of ice from open water) is an integral part of the current research.

\section{Operational Considerations}

In its current implementation, the system operates mainly within the Matlab environment. The texture feature extraction is based on the PCI modeler package. The file preparation is performed within the PCI image processing software. The segmentation phase accounts for over $80 \%$ of the total time required to analyze a single image. For a 40+ megapixel image and using two texture features, the whole process takes approximatively four hours to run on a $2.8-\mathrm{GHz}$ Intel processor. This figure is not meant as a strict reference since the time also depends on the number of egg code regions and the number of classes per region. This time frame could be significantly reduced using optimized coding since Matlab is recognized to be cumbersome for iterative procedures. This would in turn bring the system to an "operational" level in terms of processing time.

\section{Outlook}

A number of valuable output products are foreseen. The first of these is a thematic map produced at the pixel resolution (as opposed to the "homogeneous regions" used by the sea ice analysts) with three classes: ice, open water, and land. This thematic map would be a valuable complementary tool for navigation (in addition to the egg code maps). A second thematic map would retain all the sea ice classes extracted during the segmentation and classification and would be stored in a database that would be used for meteorological and ocean circulation purposes as well as global change studies. Finally, the sea ice thematic maps could instantly yield a wealth of statistics on the proportions of sea ice types and open water on a daily basis. 


\section{CONCLUSION}

This paper presents a system to classify satellite SAR data in different types of sea ice and open water. The system is based on refining a regional mapping made by ice analysts from the Canadian Ice Service to a pixel-level resolution. This approach ensures that only the proper information is extracted from the image by guiding a segmentation algorithm through the provision of the number and type of sea ice classes (and open water if present) that should be found in a particular region defined by the analyst. Likewise, the system takes advantage of a priori information to deduce which class should be assigned to a segment by a process of deduction, elimination and pattern classification. Here, this process received the name of "cognitive reasoning" because of its ability to "learn" the most likely match using minimal information.

Through two sample SAR images from the Gulf of Saint Lawrence region, being considered "difficult cases" in terms of sea ice mapping and one image of the Beaufort Sea it was shown that the MAMSEG algorithm is capable of achieving accurate segmentation with a high level of detail. The highest level of success was achieved by feeding the segmentation algorithm with a triplet of features composed of the SAR intensity and two texture features generated from the gray-level coccurrence matrix. It was also demonstrated that a "cognitive reasoning" approach was successively implemented for solving the labeling problem. Additionally, it was found that the Mahanalobis distance and the Fisher linear criterion were accurate metrics for assigning the most likely match between sea ice classes and segments produced by MAMSEG although the former was slightly superior in the overall performance. The Kolmogorov-Smirnov test was also implemented as a third metric but showed relatively poor results that were attributed to the nonuniform skewed distributions.

Problems encountered were attributed to four principal factors, two of which were generated by incomplete information on the part of the egg code themselves. The two other problems appear to have been resulting from the incomplete feature space that should include information on shape and size of the floes and some way to model the effects of the variations of the angle of incidence. Although some solutions are being considered for solving these problems, more testing is required before a refined solution is implemented.

\section{ACKNOWLEDGMENT}

The authors are thankful to the Canadian Ice Service (CIS), GEOIDE, and CRYSYS for their financial support. The CIS, as well, is thanked for providing the SAR sea ice images (all Radarsat images copyright CSA-http://www.space/gc/ca). The authors would also like to thank CIS personnel (D. Flett, R. D'Abreu, K. Wilson and M. Manore) for helpful conversations directly relating to this paper and past discussions that have supported the basis of this research. Finally, the authors which to thank the anonymous reviewers who helped improve the final version of this paper.

\section{REFERENCES}

[1] M. Manore, C. Bertoia, and H. S. Andersen, "Ice services look to the future: Assessing remote sensing needs," Backscatter, pp. 21-25, 2002.

[2] H. Deng and D. A. Clausi, "Unsupervised segmentation of synthetic aperture radar sea ice imagery using a novel Markov random field models," IEEE Trans. Geosci. Remote Sens., vol. 43, no. 3, pp. 528-538, Mar. 2005.

[3] D. Hall, "Remote sensing of snow and ice using imaging radar," in Manual of Remote Sensing, 3rd ed. New York: Wiley, 1998, vol. 2, pp. 677-703.

[4] F. Carsey, "Review and status of remote sensing of sea ice," IEEE J. Oceanic Eng., vol. 14, no. 2, pp. 127-138, Apr. 1989.

[5] D. G. Barber and E. F. LeDrew, "SAR sea ice discrimination using texture statistics: A multivariate approach," Photogramm. Eng. Remote Sens., vol. 57, no. 4, pp. 385-395, 1991.

[6] T. Agnew, R. Brown, G. Flato, H. Melling, and B. Ramsay, "Canadian contributions to GCOS-Sea ice," Atmospheric Environment Service (AES)_Environment Canada, Toronto, ON, Canada, 1999.

[7] R. Kwok and G. Cunningham, "Seasonal ice area and volume production of the Arctic Ocean: November 1996 through April 1997," J. Geophys. Res., vol. 107, no. C10, 2002.

[8] — RADARSAT Geophysical Processor System Data Users Handbook. Pasadena, CA: Jet Propulsion Lab., 2000.

[9] R. Brown and D. ONeill. (2002) National Plan for Cryospheric Monitoring-A Canadian Contribution to the Global Climate Observing System. Meteorological Service of Canada_Climate Research Branch. [Online]. Available: http://www.socc.ca/gcos_cry_plan_canada.pdf.

[10] K. C. Partington and C. Bertoia, Science Plan-Version 6.0. Washington, DC: Nat. Ice Center, 1997.

[11] O. Johannessen, E. Shalina, and M. Miles, "Satellite evidence for an arctic sea ice cover in transformation," Science, vol. 286, no. 3, pp. 1937-1939, 1999.

[12] C. Parkinson, D. Cavalieri, P. Gloersen, H. Zwally, and J. Comiso, "Arctic sea ice extents, areas, and trends, 1978-1996," J. Geophys. Res., vol. 104, no. C9, pp. 20 837-20 856, 1999.

[13] A. Nolin, F. Fetterer, and T. Scambos, "Surface roughness characterizations of sea ice and ice sheets: Case studies with MISR data," IEEE Trans. Geosci. Remote Sens., vol. 40, no. 7, pp. 1605-1615, Jul. 2002.

[14] R. Massom, Satellite Remote Sensing of Polar Regions. Boca Raton, FL: Lewis, 1991.

[15] M. Mäkynen, A. Manninen, M. Similä, J. Karvonen, and M. Hallikainen, "Incidence angle dependence of the statistical properties of $\mathrm{C}$-band $\mathrm{HH}$-polarization backscattering signatures of the Baltic sea ice," IEEE Trans. Geosci. Remote Sens., vol. 40, no. 12, pp. 2593-2605, Dec. 2002.

[16] R. Raney, "Radar fundamentals: Technical perspective," in Manual of Remote Sensing, 3rd ed. New York: Wiley, 1998, vol. 2, pp. 9-130.

[17] B. Kerman, "Information states in radar imagery of sea ice," IEEE Trans. Geosci. Remote Sens., vol. 37, no. 3, pp. 1435-1446, May 1999.

[18] C. B. Fetterer and J. Ye, "Multi-year ice concentration from Radarsat," in Proc. IGARSS, vol. 1, Aug. 3-8, 1997, pp. 402-404.

[19] L. K. Soh, C. Tsatsoulis, D. Gineris, and C. Bertoia, "ARKTOS: An intelligent system for sar sea ice classification," IEEE Trans. Geosci. Remote Sens., vol. 42, no. 1, pp. 229-248, Jan. 2004.

[20] J. A. Karvonen, "Baltic sea ice SAR segmentation and classification using modified pulse-coupled neural networks," IEEE Trans. Geosci. Remote Sens., vol. 42, no. 7, pp. 1566-1574, Jul. 2004.

[21] T. R. Reed and J. M. H. du Buf, "A review of recent texture segmentation and feature extraction techniques," Comput. Vis. Graph. Image Process., Image Understanding, vol. 57, no. 3, pp. 359-372, 1993.

[22] R. M. Haralick, K. Shanmugam, and I. Dinstein, "Textural features for image classification," IEEE Trans. Syst. Man Cybern., vol. 3, pp. 610-621, 1973.

[23] S. E. Franklin, Remote Sensing for Sustainable Forest Management. Boca Raton, FL: Lewis, 2001.

[24] P. Maillard, "Comparing texture analysis methods through classification," Photogramm. Eng. Remote Sens., vol. 69, no. 4, pp. 357-367, 2003.

[25] J. Du Buf, M. Kardan, and M. Spann, "Texture feature performance for image segmentation,” Pattern Recognit., vol. 23, no. 3/4, pp. 291-309, 1990.

[26] L. K. Soh and C. Tsatsoulis, "Texture analysis of SAR sea ice imagery using gray level co-occurrence matrices," IEEE Trans. Geosci. Remote Sens., vol. 37, no. 2, pp. 780-795, Mar. 1999. 
[27] M. E. Shokr, "Evaluation of second-order texture parameters for sea ice classification from radar images," J. Geophys. Res., vol. 96, no. C6, pp. $10625-10640,1991$.

[28] D. A. Clausi, "An analysis of co-occurrence texture statistics as a function of grey level quantization," Can. J. Remote Sens., vol. 28, no. 1, pp. $1-18,2002$

[29] — - "Comparison and fusion of co-occurrence, Gabor and MRF texture features for classification of SAR sea-ice imagery," Atmosphere-Ocean, vol. 39, no. 3, pp. 183-194, 2000.

[30] D. A. Clausi and B. Yue, "Comparing cooccurrence probabilities and Markov random fields for texture analysis of SAR sea ice imagery," IEEE Trans. Geosci. Remote Sens., vol. 42, no. 1, pp. 215-228, Jan. 2004.

[31] R. Jobanputra and D. Clausi, "Texture analysis using Gaussian weighted grey level co-occurrence probabilities," in Proc. 1st Can. Conf. Computer and Robot Vision, London, ON, Canada, May 17-19, 2004.

[32] R. Dubes and A. Jain, "Random field models in image analysis," J. Appl. Stat., vol. 16, pp. 131-164, 1993.

[33] S. Z. Li, Markov Random Field Modeling in Computer Vision. New York: Springer-Verlag, 2001.

[34] B. Tso and P. Mather, Classification Methods for Remotely Sensed Data. London, U.K.: Taylor and Francis, 2001.

[35] F. S. Cohen and D. B. Cooper, "Simple parallel hierarchical and relaxation algorithms for segmenting noncausal Markovian random fields," IEEE Trans. Pattern Anal. Mach. Intell., vol. PAMI-9, no. 2, pp. 195-219, Feb. 1987.

[36] C. S. Won and H. Derin, "Unsupervised segmentation of noisy and textured images using Markov random fields," Comput. Vis. Graphics Image Process., Graph. Models Image Process., vol. 54, no. 4, pp. 308-328, 1992.

[37] D. Geman, S. Geman, C. Graffigne, and P. Dong, "Boundary detection by constrained optimization," IEEE Trans. Pattern Anal. Mach. Intell., vol. 12, no. 7, pp. 609-628, Jul. 1990.

[38] D. K. Panjwani and G. Healey, "Markov random field models for unsupervised segmentation of textured color images," IEEE Trans. Pattern Anal. Mach. Intell., vol. 17, no. 10, pp. 939-954, Oct. 1995.

[39] Y. Dong, B. Forester, and A. Milne, "Comparison of radar image segmentation by Gaussian- and gamma-Markov random fields models," Int. J. Remote Sens., vol. 24, no. 4, pp. 711-722, 2004.

[40] S. Krishnamachari and R. Chellappa, "Multiresolution Gauss-Markov random field models for texture segmentation," IEEE Trans. Image Process., vol. 6, no. 2, pp. 251-267, Feb. 1997.

[41] G. Liu, H. Xiong, and S. Huang, "Study on segmentation and interpretation of multilook polarimetric sar images," Int. J. Remote Sens., vol. 21, no. 8, pp. 1675-1691, 2004.

[42] C. P. Lo and A. K. W. Yeung, Concepts and Techniques of Geographic Information Systems, 1st ed. ed. Upper Saddle River, NJ: PrenticeHall, 2002.

[43] R. Duda, P. Hart, and D. Stork, Pattern Classification, 2nd ed. New York: Wiley, 2001.

[44] W. Press, S. Teukolsky, W. Vettering, and B. Flannery, Numerical Recipes in C: The Art of Scientific Computing, 2nd ed. Cambridge, U.K.: Cambridge Univ. Press, 1992.

[45] D. R. Hofstadter, Gödel, Escher, Bach: An Eternal Golden Braid. New York: Basic Books, 1979

[46] L. K. Soh and C. Tsatsoulis, "Unsupervised segmentation of ers and radarsat sea ice images using multiresolution peak detection and aggregated population equalization," Int. J. Remote Sens., vol. 20, no. 15, pp. 3087-3109, 1999.

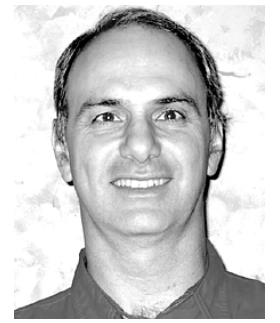

Philippe Maillard received the B.Sc. degree and M.Sc. degree in physical geography from the University of Montreal, Montreal, QC, Canada, in 1986 and 1989, respectively, specializing in remote sensing and computer mapping, and the Ph.D. degree in remote sensing and image analysis from the University of Queensland, Brisbane, Australia, in 2001

In 1988, he was offered a position at Geophysics GPR where he started a remote sensing department and remained until 1992. He then moved to Brazil to take a teaching position at the Universidade Federal de Minas Gerais, Belo Horizonte, Brazil. After completing his Ph.D. in 2001, he returned to Brazil as an Associate Professor where he remains to this day. In 2004-2005, he undertook a postdoctoral position at Systems Design Engineering, University of Waterloo, Waterloo, ON, Canada, to pursue research in sea ice classification and texture analysis. His current research interests include texture analysis for remote sensing applications, vegetation modeling using optical and SAR data, and sea ice classification from SAR data.

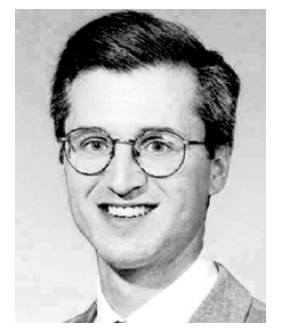

David A. Clausi (S'93-M'96-SM'03) received the B.A.Sc., M.A.Sc., and Ph.D. degrees from the University of Waterloo, Waterloo, ON, Canada, in 1990, 1992, and 1996, respectively.

After completing his doctorate, he worked in the medical imaging field at Mitra Imaging Inc., Waterloo. He started his academic career in 1997 as an Assistant Professor in geomatics engineering at the University of Calgary. In 1999, he returned to the University of Waterloo as an Assistant Professor in systems design engineering and was awarded tenure and promotion to Associate Professor in 2003. He is an active interdisciplinary and multidisciplinary researcher and has a prestigious publication record, publishing refereed journal and conference papers in the diverse fields of remote sensing, computer vision, algorithm design, and biomechanics. His primary research interest is the automated interpretation of synthetic aperture radar sea ice imagery, in support of operational activities of the Canadian Ice Service. The research results have led to successfully commercial implementations.

Dr. Clausi has received numerous graduate scholarships, conference paper awards, and a Teaching Excellence Award.

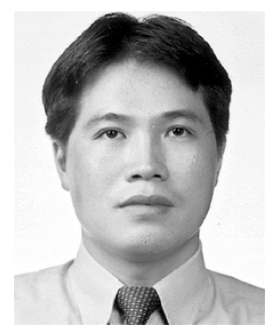

Huawu Deng (M'03) received the B.A.Sc. degree from Beijing University, Beijing, China, the M.Eng. degree from the Research Institute of Petroleum Exploration and Development, Beijing, and the Ph.D. degree from Nanyang Technological University, Singapore, in 1992, 1995, and 2001, respectively.

From 2000 to 2002, he was an Information Technology Analyst for a regional bank in Singapore. From 2002 to 2004, he was a Postdoctoral Research Fellow with the University of Waterloo, Waterloo, ON, Canada. He is currently with PCI Geomatics, Richmond Hill, ON, Canada. His research interests are in image processing (texture modeling, image classification, image segmentation, image retrieval), computer vision, pattern recognition, and remote sensing data analysis. 